\title{
Atenção integral à saúde da mulher: desafios para implementação na prática assistencial
}

Michele Pedrosa

A saúde é um direito humano fundamental que deve ser compreendida como um recurso para a vida, não sendo somente a ausência de doenças. Saúde é o resultado de um

processo de produção social que expressa a qualidade de vida de uma população, entendendo-se qualidade de vida como uma condição de existência dos homens no seu viver cotidiano, representado por um conjunto integrado de recursos sociais e pessoais.

No campo do atendimento à saúde da mulher, para que o conceito de integralidade seja efetivamente implementado faz-se necessário transformar a tradicional abordagem baseada nas características anatomo-fisiológicas do sexo feminino em uma condução direcionada para as questões referentes às peculiaridades de gênero.

Neste contexto, o setor saúde deve mover-se, então, no sentido da promoção da saúde, além das suas responsabilidades de prover serviços clínicos e de urgência. Os serviços de saúde precisam adotar uma postura abrangente que perceba e respeite as peculiaridades sociais e culturais, apoiando as necessidades individuais e comunitárias para uma vida mais saudável. A reorientação dos serviços de saúde requer mudanças na educação e no ensino dos profissionais da área de saúde, buscando induzir transformações na atitude e na organização desses serviços para que focalizem as necessidades globais do indivíduo, como pessoa integral.

Diversos desafios se colocam para a implementação da atenção integral à saúde da mulher na prática assistencial. A abordagem das questões relacionadas ao gênero feminino, presente na elaboração de diversas políticas públicas, precisa ser compreendida e incorporada por gestores e principalmente pelos profissionais de saúde. Esses profissionais, responsáveis diretos pelo atendimento à população, possuem fundamental papel na aplicação cotidiana dos conceitos de integralidade e equidade, transformando-os em ação concreta, capaz de modificar os níveis de saúde da população. 


\section{GÊNERO: CONCEITUAÇÃO, HISTORICIDADE E IMPORTÂNCIA}

\section{SANITÁRIA}

Gênero e sexo são muitas vezes considerados como sinônimos, quando na verdade correspondem a conceitos distintos. Segundo Schraiber \& D’Oliveira (1999), enquanto sexo indica uma diferença genética e anatômica inscrita no corpo, gênero indica a construção social, material e simbólica a partir desta diferença. O gênero, como elemento constitutivo das relações sociais entre homens e mulheres, é uma construção social e histórica. É construído e alimentado com base em símbolos, normas e instituições que definem modelos de masculinidade e feminilidade e padrões de comportamento aceitáveis ou não para homens e mulheres.

Segundo Martins (2004), no século XIX, com o triunfo da biologia e a ascensão do poder médico, consolidou-se um novo modo de pensar as distinções de gênero: as diferenças corporais se instalaram progressivamente como referência do feminino e do masculino. No sexo biológico, médicos, biólogos, anatomistas e fisiologistas viram a origem de uma irredutível diferença entre homens e mulheres expressada não somente na ordem físico-anatômica, como também em uma ordem moral e social. Em seu afã de definir a “mulher”, eles promoveram um intenso debate público sobre a domesticidade e a inferioridade das mulheres, a vocação maternal, a sexualidade perigosa, o pudor feminino, a pouca aptidão para a política e as ciências etc., contribuindo para fundamentar a exclusão das mulheres da esfera da cidadania e a negação de sua autonomia e subjetividade.

A constituição da obstetrícia como conhecimento prático e disciplinar, no século XIX, transforma o parto de um evento social ligado à cotidianidade das mulheres em um evento médico e hospitalar. As ciências obstétricas conferem significados práticos ao modelo biológico das diferenças sexuais, atualizando em um campo de conhecimentos práticos e disciplinares a visão da especificidade da natureza feminina e da vocação maternal da mulher.

A ginecologia também se estrutura como especialidade no século XIX, definida como a "ciência da mulher”, tomando como objeto de conhecimento e intervenção médica a sexualidade e os órgãos sexuais femininos. Com seu advento, aprofundou-se a idéia da 
diferença sexual radical das mulheres e conformou-se definitivamente um campo de práticas médicas diferenciado, para uma categoria diferenciada de clientes: as mulheres.

Para esses médicos, os órgãos sexuais determinariam a natureza nervosa, frágil e inconstante da mulher, e sua predisposição a doenças e perturbações mentais, moldando assim suas capacidades sociais. A sexualidade feminina foi representada como perigosa e paradoxal, oscilando entre a ausência de desejo - definido como a normalidade - e o desejo excessivo - fator de perturbações físicas, psíquicas e morais. Independente de como se manifestavam, procurava-se uma etiologia sexual para as doenças das mulheres, pois estas estariam inequivocamente determinadas por seu sexo.

Estes fatores concorreram para a identificação histórica e cultural do feminino como “fisiologicamente patológico”. Desta forma, o conhecimento científico relacionado ao funcionamento do corpo da mulher contribuiu de forma importante para a definição de papéis sociais distintos entre os gêneros, sendo ao homem atribuído a função de provedor e à mulher a função doméstica e reprodutiva. Estas funções foram legitimadas através de substratos biológicos relacionados a aspectos anatômicos e fisiológicos inerentes ao sexo, e por isso imutáveis.

Entretanto, nas ultimas décadas do século passado, questões de natureza sócioeconômica colaboraram na modificação dos papeis sociais relacionados ao gênero. As mulheres passaram não somente a auxiliar, como muitas vezes a ser responsável pela renda familiar. Essa atualização ideológica dos gêneros, na figura da “nova mulher independente” que controla sua fecundidade, trabalha fora e tem seu dinheiro "próprio", acabam por ocultar o aprofundamento da dupla jornada, da exploração e da forma em que estas estratégias contribuem para a reprodução da desigualdade em nível de gênero e de classe social (Giffin, 2002).

A situação de saúde envolve diversos aspectos da vida, como a relação com o meio ambiente, o lazer, a alimentação e as condições de trabalho, moradia e renda. No caso das mulheres, os problemas são agravados pela discriminação nas relações de trabalho e a sobrecarga com as responsabilidades com o trabalho doméstico. Outras variáveis como raça, etnia e situação de pobreza realçam ainda mais as desigualdades. As mulheres vivem mais do que os homens, porém adoecem mais freqüentemente. A vulnerabilidade feminina 
frente a certas doenças e causas de morte está mais relacionada com a situação de discriminação na sociedade do que com fatores biológicos.

As desigualdades sociais, econômicas e culturais se revelam no processo de adoecer e morrer das populações e de cada pessoa em particular, de maneira diferenciada. De acordo com os indicadores de saúde, as populações expostas a precárias condições de vida estão mais vulneráveis e vivem menos. O relatório sobre a situação da População Mundial (2002) demonstra que o número de mulheres que vivem em situação de pobreza é superior ao de homens, que as mulheres trabalham durante mais horas do que os homens e que, pelo menos, metade do seu tempo é gasto em atividades não remuneradas, o que diminui o seu acesso aos bens sociais, inclusive aos serviços de saúde.

A Síntese de Indicadores Sociais 2002, do IBGE, apresenta os seguintes dados: a população feminina ocupada concentra-se nas classes de rendimento mais baixas - 71,3\% das mulheres que trabalham ganham até dois salários mínimos, contra 55,1\% dos homens, e a desigualdade salarial aumenta conforme a remuneração. A proporção de homens que ganham mais de cinco salários mínimos é de 15,5\% e das mulheres é de 9,2\%. No que se refere ao trabalho doméstico, as mulheres dedicadas a essa atividade (19,2\%) e que não recebem remuneração (10,5\%) é bem maior do que a dos homens $(0,8 \%$ e 5,9\% respectivamente).

Assim, da mesma maneira que diferentes populações estão expostas a variados tipos e graus de risco, mulheres e homens, em função da organização social das relações de gênero, também estão expostos a padrões distintos de sofrimento, adoecimento e morte. Partindo-se desse pressuposto, é imprescindível a incorporação da perspectiva de gênero na análise do perfil epidemiológico e no planejamento de ações de saúde, que tenham como objetivo promover a melhoria das condições de vida, a igualdade e os direitos de cidadania da mulher.

\section{O MOVIMENTO FEMINISTA E A POLÍTICA DE ATENÇÃO INTEGRAL À SAÚDE DA MULHER}

No Brasil, a saúde da mulher foi incorporada às políticas nacionais de saúde nas primeiras décadas do século $\mathrm{XX}$, sendo limitada, nesse período, às demandas relativas à 
gravidez e ao parto. Os programas materno-infantis, elaborados nas décadas de 30, 50 e 70, traduziam uma visão restrita sobre a mulher, baseada em sua especificidade biológica e no seu papel social de mãe e doméstica, responsável pela criação, pela educação e pelo cuidado com a saúde dos filhos e demais familiares.

No âmbito do movimento feminista brasileiro, esses programas foram vigorosamente criticados pela perspectiva reducionista com que tratavam a mulher, que tinha acesso a alguns cuidados de saúde no ciclo gravídicio-puerperal, ficando sem assistência na maior parte de sua vida. Com forte atuação no campo da saúde, o movimento de mulheres contribuiu para introduzir na agenda política nacional, questões, até então, relegadas ao segundo plano, por serem consideradas restritas ao espaço e às relações privadas. Naquele momento tratava-se de revelar as desigualdades nas condições de vida e nas relações entre os homens e as mulheres, os problemas associados à sexualidade e à reprodução, as dificuldades relacionadas à anticoncepção e à prevenção de doenças sexualmente transmissíveis e a sobrecarga de trabalho das mulheres, responsáveis pelo trabalho doméstico e de criação dos filhos.

Durante seus primeiros anos de atuação política, o movimento feminista buscou construir uma base organizacional apoiado em grupos autônomos e, ao mesmo tempo, expandir seu alcance levando suas propostas a organizações de classe, sindicatos e universidades. Uma das reivindicações do movimento feminista consistia no direito da mulher ao controle de seu corpo, permitindo um exercício da sexualidade livre da ameaça da gravidez não desejada, expressando uma nova consciência do corpo feminino, e uma busca das transformações das questões de gênero.

As mulheres organizadas argumentavam que as desigualdades nas relações sociais entre homens e mulheres se traduziam também em problemas de saúde que afetavam particularmente a população feminina. Por isso, fazia-se necessário criticá-los, buscando identificar e propor processos políticos que promovessem mudanças na sociedade e conseqüentemente na qualidade de vida da população.

Com base naqueles argumentos, foi proposto que a perspectiva de mudança das relações sociais entre homens e mulheres prestasse suporte à elaboração, execução e avaliação das políticas de saúde da mulher. As mulheres organizadas reivindicaram, 
portanto, sua condição de sujeitos de direito, com necessidades que extrapolavam o momento da gestação e parto, demandando ações que lhes proporcionassem melhoria das condições de saúde em todas os ciclos de vida. Ações que contemplassem as particularidades dos diferentes grupos populacionais, e as condições sociais, econômicas, culturais e afetivas, em que estivessem inseridos.

As primeiras experiências de elaboração de políticas públicas com perspectiva de gênero ocorreram na década de 80. Particularmente relevante foi a articulação entre as universidades, o movimento feminista e o Ministério da Saúde que veio a resultar no Programa de Assistência Integral à Saúde da Mulher (PAISM). O PAISM foi também influenciado pelos movimentos sanitaristas, principalmente na adoção de uma perspectiva integral de saúde e defesa da universalidade. Divulgado em 1983, representava um raro exemplo de colaboração entre Estado e sociedade civil, constituindo em uma das primeiras iniciativas governamentais de incorporação de princípios feministas em políticas públicas de saúde.

O PAISM adotou uma atitude revolucionária ao enfocar a saúde da mulher de forma integral, fugindo da abordagem limitada à saúde reprodutiva, e abrangendo tudo aquilo que era preconizado em termo de desenvolvimento de políticas e programas setoriais eqüitativos em termo de gênero. No entanto, enormes dificuldades vêm sendo enfrentadas para implantar efetivamente a integralidade e a equidade no interior de um sistema de saúde que tende a seguir um modelo de especialização na clínica e verticalidade dos programas. Por outro lado, assim como ocorreu em relação ao Sistema Único de Saúde (SUS), ao ser circunscrito a uma proposta assistencial, o PAISM não se consubstanciou como projeto de saúde de âmbito multisetorial.

O processo de implantação e implementação do PAISM apresentou especificidades no período de 84 a 89 e na década de 90, sendo influenciado, a partir da proposição do SUS, pelas características da nova política de saúde, pelo processo de municipalização e principalmente pela reorganização da atenção básica, por meio da estratégia do Programa Saúde da Família. Estudos realizados para avaliar os estágios de implementação da política de saúde da mulher demonstram a existência de dificuldades na implantação dessas ações e, 
embora não se tenha um panorama abrangente da situação em todos os municípios, pode-se afirmar que a maioria enfrenta ainda dificuldades políticas, técnicas e administrativas.

Alem disso, a organização e financiamento precários deste programa não têm permitido um desempenho satisfatório tanto no atendimento às necessidades básicas no ciclo gravídicio-puerperal, quanto na assistência a outros problemas de saúde. Como conseqüência observa-se um relativo esvaziamento dos seus aspectos mais criativos e inovadores, reduzindo-o, na maioria das vezes, a metas focalizadas e à assistência ginecoobstétrica tradicional.

Com o objetivo de consolidar os avanços no campo dos direitos sexuais e reprodutivos o Ministério da Saúde publicou em 2004 “A Política Nacional de Atenção Integral à Saúde da Mulher” que incorpora num enfoque de gênero, a integralidade e a promoção da saúde como princípios norteadores. As ações de saúde propostas por esta política enfatizam: a melhoria da atenção obstétrica, o planejamento familiar, a atenção ao abortamento inseguro, o combate à violência doméstica e sexual e o cuidado a saúde da adolescente e da mulher no climatério. Agrega, também, a prevenção e o tratamento de mulheres vivendo com HIV/AIDS, as portadoras de doenças crônicas não transmissíveis, incluindo saúde mental, e de câncer ginecológico. Além disso, amplia as ações para grupos historicamente alijados das políticas públicas (lésbicas, mulheres negras, indígenas, residentes em zonas rurais e mulheres em situação de prisão), nas suas especificidades e necessidades. Esta política ainda propõe diretrizes para a humanização e a qualidade do atendimento, questões ainda pendentes na atenção à saúde das mulheres.

\section{O SISTEMA DE SAÚDE E A ATENÇÃO À MULHER}

Classicamente, assistência à saúde da mulher refere-se ao atendimento de condições de saúde relacionadas às funções reprodutivas, sobretudo o atendimento ao ciclo gravídiciopuerperal e a anticoncepção, sendo ainda incluídos o controle do câncer de colo uterino e mama. Entretanto, as principais causas de contato das mulheres com o sistema de saúde são de natureza não-reprodutivas, com destaque para as doenças crônicas. No Brasil, segundo o Sistema de Informação de Mortalidade (2003), as principais causas de morte da população feminina são as doenças cardiovasculares, destacando-se o infarto agudo do miocárdio e o 
acidente vascular cerebral; as neoplasias, principalmente o câncer de mama, de pulmão e o de colo do útero; as doenças do aparelho respiratório, marcadamente as pneumonias; doenças endócrinas, nutricionais e metabólicas, com destaque para o diabetes; e as causas externas.

Baseando-se na concepção reprodutiva, foram estruturados serviços específicos para o atendimento à saúde da mulher, o que auxiliou na facilitação do acesso aos cuidados com a saúde reprodutiva. Estes serviços, entretanto, acabaram por se contrapor ao princípio da abordagem integral, contribuindo para a fragmentação no atendimento à mulher, visto que questões de ordem não reprodutiva dificilmente são avaliadas durante este contato com o sistema.

Por outro lado, o atendimento à mulher por condições não relacionadas à reprodução, sejam agudas ou crônicas, carece da abordagem de gênero. Não há, rotineiramente, preocupações com características próprias da mulher, sejam de ordem biológica, psicológica, cultural ou social. Na maioria das vezes, nem mesmos questões relativas às diferenças anátomo-fisiológicas são consideradas.

$\mathrm{O}$ atendimento fragmentado, baseado nas alterações biológicas e centrado em intervenções tecnológicas, não se limita ao atendimento à mulher, sendo constitutivo do modelo biomédico predominante na assistência à saúde. A interferência na saúde da mulher dessas características do modelo assistencial reflete-se nas respostas insuficientes às necessidades de saúde, enfrentadas ainda de forma indistinta entre mulheres e homens, estes historicamente considerados como o padrão de normalidade.

Outro aspecto do sistema de saúde a ser considerado, que apesar de não se restringir à saúde da mulher tem importante influencia na abordagem deste grupo populacional, é a comercialização da saúde, própria das economias de mercado e sua capacidade de definir necessidades. As lucrativas indústrias de equipamentos e fármacos relacionam-se com a saúde como um bem de consumo, a ser adquirido a custos cada vez mais elevados. Iniciando-se antes do nascimento e estendendo-se até a velhice e a morte, a mulher é objeto permanente de promoção de consumo de bens e serviços que afirmam visar a promoção da sua saúde, beleza, sexualidade e felicidade geral (Barros, 1991 apud Silver,1999). 
Assim, a oferta e utilização de tecnologias médicas são orientadas pelos interesses de mercado e não por razões de interesse sanitário. Esta tentativa de medicalizar a vida da mulher é altamente lucrativa, legitimada em ampla, e enviesada, literatura científica, que precisa ser avaliada cuidadosamente. Os profissionais de saúde tem papel fundamental na afirmação deste cenário, e como conseqüência de uma formação profissional acrítica, incorporam, indiscriminadamente, a sua prática, tecnologias em saúde.

A terapia de reposição hormonal (TRH) é um dos vários exemplos que ilustram como necessidades tecnológicas, definidas pelas grandes indústrias da saúde, foram aceitas como verdades sanitárias. Através de intenso trabalho de propaganda entre o publico leigo e entre os profissionais médicos, as indústrias farmacêuticas difundiram a idéia que a menopausa constitui um estado patológico, que como tal necessitaria de tratamento medicamentoso de longo prazo.

A equidade é um importante ponto a ser destacado na discussão do atendimento à mulher, visto existir diferenças nítidas entre mulheres de diferentes classes sociais no que se relaciona a vários aspectos da assistência, sobretudo ao acesso a serviços e insumos. Apesar da transformação das práticas e ideologias dominantes, o gênero é um sistema entre outros que atuam de forma interlaçada no plano social, com resultados às vezes contraditórios, diferentes para mulheres em variadas situações sociais, econômicas e culturais (Giffin, 2002).

Na discussão da assistência à saúde da mulher é fundamental avaliar a qualidade do atendimento nos serviços de saúde, determinando a capacidade destes serviços em contemplar todas as condições de saúde com ações de promoção, prevenção, assistência e recuperação, assegurando atenção integral e individualizada. Garantir acesso à consulta pode não ser suficiente, pois em muitas vezes a qualidade da atenção é tão precária que em pouco ou nada contribuem para melhoria da saúde da mulher.

Correa \& Petchesky (1994, apud Silver, 1999) propuseram quatro princípios para constituir as bases éticas dos direitos reprodutivos e sexuais:

- $\quad$ o respeito à integridade corporal, entendido como o direito de segurança e controle sobre o próprio corpo e incorporando o princípio da integralidade; 
- $\quad$ o respeito à pessoa, a seus desejos e suas experiências, considerando a mulher como a responsável pelas tomadas de decisão em relação à sua saúde;

- $\quad$ a igualdade, aplicando-se tanto nas diferenças homem e mulher, como entre mulheres de diferentes classes social, idade, nacionalidade ou etnicidade;

- $\quad$ o respeito as diversidades - as diferenças entre as mulheres em valores, cultura, desejos reprodutivos, religião, orientação sexual, condição familiar ou médica.

Estes princípios têm uma clara aplicabilidade para a saúde da mulher em geral, no que tange tanto aos problemas não reprodutivos como aos problemas reprodutivos e sociais. A incorporação desses conceitos na pratica cotidiana dos serviços certamente contribuiria para melhoria da assistência, com conseqüente impacto positivo na qualidade de vida das mulheres. 


\section{O PROFISSIONAL DE SAÚDE E O ATENDIMENTO INTEGRAL À SAÚDE DA MULHER}

A experiência demonstra que é necessário não apenas ter boas intenções e um programa normativamente correto, como o PAISM, para garantir atendimento de qualidade à mulher. É preciso desenvolver, cada vez mais, a capacidade de gerenciar os serviços e formar profissionais da saúde orientados a uma assistência integral e humana, com equidade, e sem o uso abusivo de tecnologias médicas.

Entretanto, a formação dos profissionais de saúde, carece, em sua maioria de conteúdos relacionados a aspectos não biológicos da saúde. No que se refere especificamente à formação médica, nota-se o predomínio da lógica cartesiana mecanicista e dos conceitos flexnerianos, e a não incorporação de saberes provenientes das ciências sociais e humanas relacionadas à saúde. Os profissionais assim formados não se apresentam sensibilizados nem suficientemente instrumentalizados para lidar com as múltiplas dimensões do processo saúde-doença, dificultando de forma determinante a mudança de um modelo biologicista e fragmentado para uma atenção holística e integral.

O Ministério da Saúde e o Ministério da Educação instituíram em 2002 o Programa Nacional de Incentivo a Mudanças Curriculares nos Cursos de Medicina (Promed) com o objetivo de incentivar as escolas médicas a incorporar mudanças pedagógicas significativas nos currículos dos cursos de medicina. O Promed apresenta uma proposta de intervenção no processo formativo para que os programas de graduação possam deslocar o eixo da formação médica - centrado na assistência individual prestada em unidades hospitalares para um outro em que a formação esteja sintonizada com o SUS, em especial com a atenção básica - considerando as dimensões sociais, econômicas e culturais que se fazem presentes no processo de adoecimento humano -, no sentido de preparar os profissionais para enfrentar os problemas do binômio saúde-doença da população nas esferas familiar e comunitária, e não apenas na instância dos serviços.

Entretanto, observam-se algumas incongruências neste sentido, ao se analisar diretrizes traçadas pelas mesmas instâncias gestoras para a formação médica. A análise da resolução do Conselho Nacional de Residência Médica (2004), órgão do Ministério da Educação, que dispõe sobre conteúdos do Programa de Residência Médica de Obstetrícia e 
Ginecologia demonstra a manutenção dos conceitos dominantes em relação tanto à formação médica quanto a abordagem à saúde da mulher. Os aspectos biológicos, com destaque para as questões reprodutivas da saúde da mulher, são predominantes, senão exclusivos. Questões relativas aos aspectos sociais, culturais e psicológicos, assim como discussões sobre gênero são negligenciadas.

Desta forma, como conseguir que os princípios norteadores do PAISM sejam implementados na prática assistencial? Não há programa, independente de sua excelência teórica e técnica, que consiga êxito em um contexto marcado por baixo investimento de ordem estrutural e processual, além de recursos humanos com formação conceitual não consonante com os princípios de integralidade propostos.

\section{CONCLUSÃO}

A implementação das políticas de atenção integral à saúde da mulher é um desafio posto para o sistema de saúde. Estas políticas apresentam caráter contra-hegemônico à medida que introduzem conceitos de assistência à saúde que se contrapõem a maneira histórica de se compreender o processo saúde-doença: sexo x gênero, biologismo x holismo, integralidade x fragmentação.

A mudança de modelo de assistência não depende somente da construção de planos e programas. Faz-se necessária a incorporação dos princípios propostos na prática cotidiana dos serviços, que devem contar com suficientes recursos financeiros e com recursos humanos capacitados a executar este novo modelo.

Estes desafios somente serão superados com a articulação dos diversos segmentos sociais envolvidos na questão. Estado, profissionais de saúde e usuárias precisam trabalhar de maneira colaborativa no desenvolvimento de estratégias que induzam a reorganização da atenção à saúde da mulher no sentido da integralidade e equidade. 


\section{BIBLIOGRAFIA}

Bello W., 2004. O dinheiro e a doença. Disponível em http://www.planetaportoalegre.net

Borges S M N, 1991. Propostas Para Uma Relação:- Profissionais de Saúde e Mulheres. Cadernos de Saúde Publica, 7(2): 284-289.

BRASIL. Ministério da Saúde. Secretaria de Atenção à Saúde, 2004. Política nacional de atenção integral à saúde da mulher: princípios e diretrizes. Brasília: Ministério da Saúde, 82 p.

GIFFIN, K, 1991. Mulher e Saúde. Cadernos de Saúde Publica, 7(2):133-134.

GIFFIN, K, 1991. Nosso corpo nos pertence: a dialética do biológico e do social. Cadernos de Saúde Publica, 7(2):190-200.

GIFFIN, K., 2002. Pobreza, desigualdade e eqüidade em saúde: considerações a partir de uma perspectiva de gênero transversal. Cadernos de Saúde Pública, 18(S):103-112.

Homedes N.\& Ugalde A., 2005. Why neoliberal health reforms have failed in Latin America. Health Policy 71, 83-96

IBGE. Estimativas de população para o Brasil em 2003. Brasília: Ministério da Saúde.

MARTINS, A.P.V., 2004. Visões do Feminino, a medicina da mulher nos séculos XIX e XX. Editora Fiocruz. Rio de Janeiro.

Ministério da Educação. Conselho Nacional de Residência Medica. Resolução CNRM N ${ }^{\circ}$ 16/2004. Dispõe sobre conteúdos do Programa de Residência Médica de Obstetrícia e Ginecologia. Brasília: MEC. 
Ministério da Saúde, 2002. Uma nova escola médica para um novo sistema de saúde. Saúde e Educação lançam programa para mudar o currículo de medicina. Revista de Saúde Pública,36(3):375-8.

MITJAVILA, M. e ECHEVESTE, L., 1994 - Sobre a Construção Social do Discurso Médico em Torno da Maternidade. IN: Alternativas Escassas: Saúde, Sexualidade e Reprodução na América Latina. São Paulo: PRODIR/FCC, Rio de Janeiro.

OSIS, MJMD, 1998. Paism: um marco na abordagem da saúde reprodutiva no Brasil. Cadernos de Saúde Publica,14(Supl. 1):25-32.

PITANGUY JO. Movimento Nacional e Internacional de Saúde e Direitos Reprodutivos. In: Giffin K, Costa SH. Questões da saúde reprodutiva. Rio de Janeiro: FIOCRUZ; 1999.

SCHRAIBER, L.B. \& D'OLIVEIRA, A. F.L.P., 1999. Violência contra mulheres: Interfaces com a Saúde. Interface, Comunicação, Educação, vol 3, n. 5.

SILVER, L. D. "Direito à Saúde ou Medicalização da Mulher? Implicações para a avaliação dos Serviços de Saúde para Mulheres. In: Giffin, K. e Costa, S.H. (orgs). Questões da Saúde Reprodutiva. Rio de Janeiro: FIOCRUZ, 1999 (299-317).

UNFPA- United Nation Population Fund, 2005. Relatório sobre a situação da população mundial. Disponível em: < www.unfpa.org >. 